\title{
Author Correction: Accelerated antimicrobial discovery via deep generative models and molecular dynamics simulations
}

Payel Das (1), Tom Sercu, Kahini Wadhawan, Inkit Padhi, Sebastian Gehrmann, Flaviu Cipcigan, Vijil Chenthamarakshan, Hendrik Strobelt, Cicero dos Santos, Pin-Yu Chen, Yi Yan Yang (D), Jeremy P. K. Tan, James Hedrick, Jason Crain (D) and Aleksandra Mojsilovic

Correction to: Nature Biomedical Engineering https://doi.org/10.1038/s41551-021-00689-x, published online 11 March 2021.

In the version of this Article originally published, the competing interests statement incorrectly stated "The authors declare no competing interests.", but the statement should have read as below; all versions of the Article have been amended.

"The authors have filed the following patent applications related to this work: (1) application no. US2021/01025A1 on the CLaSS method for generating attribute-based samples (inventors: P.D., T.S., K.W., C.d.S., I.P. and S.G.); (2) application no. 16/880021 on the filtering of AI-designed molecules for laboratory testing (inventors: P.D., F.C., K.W., I.P., V.C., P.-Y.C., A.M., T.S. and C.d.S.); and (3) application no. 16/880280 on AI-designed antimicrobial peptides (inventors: P.D., F.C., J.H., Y.Y.Y., K.W., I.P., V.C. and J.P.K.T.).”

Published online: 28 June 2021

https://doi.org/10.1038/s41551-021-00771-4

(๑) The Author(s), under exclusive licence to Springer Nature Limited 2021 